\title{
Identifying Significant In-Game Statistics and Developing Prediction Models for Outcomes of NCAA Division 1 Football Championship Subdivision (FCS) Games
}

\author{
Rhonda C. Magel and Joseph C. Long \\ Department of Statistics, North Dakota State University, Fargo, ND 58108, USA
}

\begin{abstract}
Models are developed which estimate point spread of FCS Division 1 college football games, and the probability that a particular team will win the football game when the in-game statistics are known. These models are then used to predict the team that will win a future game when the differences between the in-game statistics are estimated before the game. One of the models increased the estimated prediction percentage by $7.7 \%$ over using only the team rankings for the random sample of 52 games selected played during the 2012 year.
\end{abstract}

Keywords: logistic regression; OLS regression; Odds ratios; point spread; probability of winning

\section{Introduction}

In this research, we will focus on finding the in-game statistics that are most significant in determining whether or not a given team will win the football game. Our focus will be on only NCAA Division 1 Football Championship (FCS) teams. The NCAA does have other football divisions, including Division 2, Division 3, and Division 1 Football Bowl Subdivision (FBS). The FCS is unique in that teams in this Subdivision may end up playing both FBS teams and Division 2 teams. We will begin by mentioning some of the past research on football that has been done in this area.

Harville (1977) studied home field advantage of college football teams. He found this translated to the home team having a 3.4 point advantage over the away team after sampling 1,024 games consisting mainly of Division 1 teams.

Willoughby (2002) conducted a study on the Canadian Football League using logistic regression in order to identify factors leading to a team's overall success. Willoughby categorized teams into "very good" teams, "average" teams, and "poor" teams. He tested his models using known in-game statistics and found that when these were known, the models predicted $85.9 \%$ correctly for "very good" teams, $90.2 \%$ correctly for "average" teams, and $78.8 \%$ correctly for "poor" teams.

Magel and Childress (2012) conducted an analysis of games played by the National Football League (NFL) and analyzed the outcome effects based on the turnover margin. They sampled 1,783 regular season games from the 2001 to the 2007 seasons and fit a logistic regression model based on the turnover margin of the

Rhonda C. Magel and Joseph C. Long, Department of Statistics, North Dakota State University, Fargo, ND 58108, USA. Email: 


\section{IDENTIFYING SIGNIFICANT IN-GAME STATISTICS AND DEVELOPING PREDICTION \\ MODELS FOR OUTCOMES OF NCAA DIVISION 1 FOOTBALL CHAMPIONSHIP SUBDIVISION (FCS) GAMES}

games. They used the model that they developed on the 2008 NFL regular season games and found that knowing the turnover margin of a team and whether or not that team was playing at home, they correctly predicted $72.16 \%$ of the outcomes of the games.

In this research, we develop models using least squares regression and logistic regression

to predict the point spread of a game and to estimate the probability of a given team winning a game, respectively, based on knowing the values of the significant in-game statistics. We will then try to use these models in predicting the outcomes of future games in which the in-game statistics are not known in advance.

\section{Collection of Data}

Data was collected from three regular seasons of the NCAA Division 1 FCS games played in 2009, 2010, and 2011 using a stratified random sample. Sampling consisted of randomly selecting five out of thirteen conferences in the NCAA Division 1 FCS. Within each of the five conferences, a random sample of half of the teams in the conference was selected. For each year and each team, four regular season games were randomly sampled from game 1 through game 11 and the in-game statistics for the selected games were collected. Due to the fact that the sampling of games was based on individual teams, there was a problem of sampling the same game more than once. When this happened, the following game that the selected team played was used for the sample. The sampled games are shown in Table 1 along with their respective conference, team, and year. In total, 228 games were sampled.

In-game statistics from each of the randomly sampled games were collected from two websites, ESPN College Football Score Board (ESPN, 2013) and the official NCAA Football Statistics Database (NCAA, 2013). The following in-game statistics were obtained from the ESPN College Football Score Board website (ESPN, 2013) for both of the teams playing: points scored; number of first downs; third down efficiency, total yards, rushing yards, passing yards, pass completions, pass attempts, rushing attempts, penalties, turnovers, possession time, punt returns, punt return yards, and whether the team was playing home or away. The NCAA Football Statistics Database (NCAA, 2013) was used to collect the number of sacks a team's defense had during the game.

Differences were calculated between the in-game statistics for the two teams playing in the football game in the order of, "team of interest" statistics minus "opposing team" statistics. The following differences were considered as possible independent variables to be entered in both the least squares model and the logistic model to estimate the point spread of a game and the probability of the "team of interest" winning the game, respectively:

- The difference between times of possession (diff_top);

- The difference between offensive yards (diff_yards);

- The difference between rushing yards (diff_rushyards);

- The difference between passing yards (diff_passyards);

- The difference between number of interceptions (diff_int);

- The difference between number of fumbles (diff_fumble);

- The difference between number of turnovers, turnover margin (TOM);

- The difference between number of penalties (diff_pen);

- The difference between penalty yards (diff_pen_yards); 
- The difference between probabilities of pass completions (diff_pass_comp);

- The difference between number of defensive sacks (diff_sacks);

- The difference between number of first downs (diff_first);

- The difference between third down conversion probabilities (diff_3rd_pct);

- The difference between punt returns (diffpunt_return);

- The difference between punt return yards (diff_puntyards);

- The difference between the ratio of punt return yards over punt returns (diffave_puntyards);

- The difference between average yards per offensive play (diffave_yardsperplay)

Table 1

Games Sampled for Model Fitting

\begin{tabular}{|c|c|c|c|}
\hline \multicolumn{4}{|c|}{ Big South Conference } \\
\hline Team & 2009 Games & 2010 Games & 2011 Games \\
\hline Charleston Southern & $3,5,7,9$ & $2,7,8,10$ & $2,4,7,10$ \\
\hline Coastal Carolina & $3,4,7,11$ & $1,4,7,11$ & $2,4,6,9$ \\
\hline Gardner-Webb & $3,9,10,11$ & $4,5,9,11$ & $1,2,5,6$ \\
\hline \multicolumn{4}{|c|}{ Ohio Valley Conference } \\
\hline Team & 2009 Games & 2010 Games & 2011 Games \\
\hline Eastern Illinois & $2,3,9,11$ & $2,5,7,11$ & $4,7,9,10$ \\
\hline Eastern Kentucky & $1,3,6,9$ & $3,7,9,10$ & $3,6,7,11$ \\
\hline Jacksonville State & $1,2,4,8$ & $3,4,7,11$ & $2,7,8,10$ \\
\hline Tennessee-Martin & $3,6,8,10$ & $1,5,6,10$ & $4,8,9,10$ \\
\hline \multicolumn{4}{|c|}{ Patriot League } \\
\hline Team & 2009 Games & 2010 Games & 2011 Games \\
\hline Bucknell & $4,6,8,9$ & $1,2,6,8$ & $4,6,7,11$ \\
\hline Fordham & $1,3,9,10$ & $1,3,6,10$ & $3,4,8,10$ \\
\hline GeorgeTown & $3,4,5,11$ & $2,4,7,11$ & $2,4,7,8$ \\
\hline \multicolumn{4}{|c|}{ Southem Conference } \\
\hline Team & 2009 Games & 2010 Games & 2011 Games \\
\hline Chattanooga & $1,2,9,11$ & $1,2,8,9$ & $1,4,5,9$ \\
\hline Elon & $2,3,9,11$ & $1,3,8,10$ & $1,6,8,9$ \\
\hline Georgia Southern & $1,6,8,10$ & $1,3,4,6$ & $1,4,6,11$ \\
\hline Samford & $1,2,4,6$ & $2,3,7,11$ & $2,6,10,11$ \\
\hline \multicolumn{4}{|c|}{ Southwestem Athletic Conference } \\
\hline Team & 2009 Games & 2010 Games & 2011 Games \\
\hline Alabama State & $3,7,8,10$ & $3,4,5,10$ & $2,6,7,9$ \\
\hline Alcorn State & $1,7,9,11$ & $5,7,9,11$ & $3,5,9,11$ \\
\hline Grambling State & $2,4,6,11$ & $4,5,6,11$ & $1,4,8,9$ \\
\hline Jackson State & $1,3,8,11$ & $1,3,4,6$ & $2,3,7,11$ \\
\hline Southern University & $3,8,9,11$ & $2,5,6,11$ & $4,5,7,11$ \\
\hline
\end{tabular}


In addition to the in-game statistic differences mentioned, we also obtained the rank that each team had just before playing the given game based on ranks collected from the website CompughterRatings.com (CompughterRatings.com, 2013). The website, developed by Steve Pugh, gives ranks of college football teams from every division based on a model using past performance measures of teams. The theory behind the set of ranks given by Steve Pugh is based on linear model theory and is given on the website. The difference in ranks between the two teams playing in every game was calculated.

\section{Model Development}

The stepwise logistic regression technique was initially used to develop a model for estimating the probability that the "team of interest" would win the football game. The dependent variable, $\mathrm{Y}$, in this model was equal to " 1 " if the "team of interest" won the game, and " 0 " if the "team of interest" lost the game. The variables considered for possible inclusion in the model were the differences between the in-game statistics mentioned in Section 2 for both of the teams in the game and the indicator variable for the "team of interest" indicating whether or not the game was being played at home. We began by using an $\alpha$ level of 0.25 for entry and of 0.20 for exit into the initial model. Variables were then excluded from the initial model, one at a time, which were not significant at $\alpha$ equal to 0.05 . The first variable removed was the variable with the largest $\mathrm{p}$-value if the $\mathrm{p}$-value were greater than 0.05 . The model was refitted after each variable was removed from the model and the remaining variables were tested for significance. If the variable associated with the largest p-value was not significant in the model at $\alpha$ equal to 0.05 , it was removed and the process continued, otherwise, the process stopped. The final variables included in this model (Model 1) were the following: turnover margin; difference in number of defensive sacks, difference in pass completion probabilities, difference in third down conversion probabilities, difference in number of punt returns, and difference in average yards per offensive play. No intercept term was used in the logistic transformed model since theoretically if both teams had the same in-game statistics, the game should end in a tie and the intercept should be zero, with the model giving a 0.50 probability to the team of interest winning the game. The Hosmer and Lemeshow Goodness-of-Fit test was used to test the hypothesis that the logistic regression model was a good fit. The p-value of this test was found to be 0.9663 indicating that the logistic regression model should be appropriate. The parameter estimates for Model 1 and associated test statistics for each of the variables in the model are given in Table 2. Table 3 gives the corresponding odds ratios and confidence intervals for the parameters associated with each of the variables in the model.

The turnover margin (TOM) for the "team of interest" has an odds ratio estimate of 2.631. This indicates that when the turnover margin increases by 1 for the "team of interest", this team is 2.631 times more likely to win the game. The odds ratio estimate for the difference in average number of yards per offensive play was equal to 1.649, indicating if the difference in average numbers of yards per play increased by 1 for the "team of interest", the odds of that team winning the game get multiplied by 1.649. The odds ratio for the difference in $3^{\text {rd }}$ down conversion probabilities is high because a one unit increase in this case would be going from a $3^{\text {rd }}$ down conversion probability difference of 0 to a third down conversion probability difference of 1 (this would indicate the "team of interest" was converting all of their $3^{\text {rd }}$ downs and the "opposing team" was converting none of theirs). If this happened, the odds of a team winning the game would get multiplied by 160.158 . A team increasing their $3^{\text {rd }}$ down conversion probability by 0.10 , over another team would have the effect of increasing 
their odds of winning by approximately 16 . The odds ratio for differences in pass completion probabilities is also high because a one unit increase in this case would mean that a team went from a $0 \%$ difference of percentage of pass completions with another team to a $100 \%$ difference of pass completions (meaning the "team of interest" was getting all of its passes and the "opposing team" was not completing any of their passes). If a team increased their pass completion percentage difference by 0.10 over another team, the estimated odds of this team winning the game would get multiplied by 58.75. Model 1 did not end up including the indicator variable for whether the team was playing at home. Past research has shown that home field advantage is significant in determining the outcome for football games (Harville (1977)). We did place the home indicator variable into Model 1, estimated the coefficient, and tested whether or not this indicator variable was significant. Results are given in Table 4. The estimated coefficient for the home indicator variable was negative, indicating that playing at home would have a negative impact on a team, going against the research by Harville (1977). The home indicator variable was also found to not be significant ( $\mathrm{p}$-value $=0.3921$ ). It appears that home advantage is explained by some of the in-game statistic variables already in the model. The home indicator variable was taken out of the model.

Table 2

Parameter Estimates for Model 1

\begin{tabular}{|cccccc|}
\hline Parameter & \multirow{2}{*}{ DF } & Estimate & $\begin{array}{c}\text { Standard } \\
\text { Error }\end{array}$ & $\begin{array}{c}\text { Wald } \\
\text { Chi-Square }\end{array}$ & \multirow{2}{*}{ Pr > ChiSq } \\
\hline TOM & 1 & 0.9673 & 0.1825 & 28.1057 & $<.0001$ \\
diff_pass_comp & 1 & 6.3759 & 1.8778 & 11.5295 & 0.0007 \\
diff_sacks & 1 & 0.6768 & 0.1565 & 18.7002 & $<.0001$ \\
diff_3rd_pct & 1 & 5.0762 & 1.5456 & 10.7868 & 0.001 \\
diffpunt_return & 1 & 0.5857 & 0.1689 & 12.0194 & 0.0005 \\
diffave_yardsperplay & 1 & 0.5001 & 0.1744 & 8.221 & 0.0041 \\
\hline
\end{tabular}

Table 3

Odds Ratios for Model 1

\begin{tabular}{|cccc|}
\hline Effect & $\begin{array}{c}\text { Odds Ratio Estimates } \\
\text { Point Estimate }\end{array}$ & \multicolumn{2}{c|}{$\begin{array}{c}\text { 95\% Wald } \\
\text { Confidence Limits }\end{array}$} \\
\hline TOM & 2.631 & 1.84 & 3.762 \\
diff_pass_comp & 587.532 & 14.814 & $>999.999$ \\
diff_sacks & 1.968 & 1.448 & 2.674 \\
diff_3rd_pct & 160.158 & 7.744 & $>999.999$ \\
diffpunt_return & 1.796 & 1.29 & 2.501 \\
diffave_yardsperplay & 1.649 & 1.171 & 2.321 \\
\hline
\end{tabular}


A second logistic regression model was developed (Model 2) to estimate the probability that the "team of interest" would win the football game. In addition to all the differences of the in-game statistics included in Model 1, this model also contained the difference in ranks of the two teams just before the game was played. The ranks were taken from the website CompughterRatings.com (2013). Difference in ranks was found to be significant ( $\mathrm{p}$-value $=0.0005$ ). The parameter estimates and odds ratios for this model are given in Tables 5 and 6 , respectively. It is noted that if the differences in ranks between the two teams playing increase by 1 , the odds of the "team of interest" winning the game are multiplied by approximately 1.008 .

Table 4

Addition of Home into Model 1

\begin{tabular}{|cccccc|}
\hline Parameter & DF Estimate & $\begin{array}{c}\text { Standard } \\
\text { Error }\end{array}$ & $\begin{array}{c}\text { Wald } \\
\text { Chi-Square }\end{array}$ & \multirow{2}{*}{ Pr > ChiSq } \\
\hline Home & 1 & -0.3329 & 0.389 & 0.7324 & 0.3921 \\
TOM & 1 & 0.9748 & 0.1828 & 28.4259 & $<.0001$ \\
diff_pass_comp & 1 & 6.696 & 1.9175 & 12.1941 & 0.0005 \\
diff_sacks & 1 & 0.6936 & 0.1595 & 18.9165 & $<.0001$ \\
diff_3rd_pct & 1 & 5.1684 & 1.5829 & 10.6613 & 0.0011 \\
diffpunt_return & 1 & 0.5966 & 0.1718 & 12.0511 & 0.0005 \\
diffave_yardsperplay & 1 & 0.503 & 0.175 & 8.2632 & 0.004 \\
\hline
\end{tabular}

Table 5

Parameter Estimates for Model 2

\begin{tabular}{|cccccc|}
\hline Parameter & \multirow{2}{*}{ DF Estimate } & $\begin{array}{c}\text { Standard } \\
\text { Error }\end{array}$ & $\begin{array}{c}\text { Wald } \\
\text { Chi-Square }\end{array}$ & \multirow{2}{*}{ Pr $>$ ChiSq } \\
\hline Difference_in_rank & 1 & 0.00824 & 0.00235 & 12.2622 & 0.0005 \\
TOM & 1 & 1.1234 & 0.2223 & 25.54 & $<.0001$ \\
diff_pass_comp & 1 & 7.5924 & 2.1403 & 12.5843 & 0.0004 \\
diff_sacks & 1 & 0.7363 & 0.1774 & 17.222 & $<.0001$ \\
diff_3rd_pct & 1 & 4.8726 & 1.6103 & 9.1565 & 0.0025 \\
diffpunt_return & 1 & 0.6185 & 0.1955 & 10.0089 & 0.0016 \\
\hline diffave_yardsperplay & 1 & 0.4522 & 0.2085 & 4.7029 & 0.0301 \\
\hline
\end{tabular}

Model 3 used ordinary least squares regression techniques to develop a model to estimate the point spread of the game in the order points for the "team of interest" minus points for the "opposing team". Model 3 was formed by beginning with the stepwise selection technique at an $\alpha$ level of 0.25 for entry and 0.20 for exit from the model using the variables in Table 1 for possible entry into the model along with the home indicator 
IDENTIFYING SIGNIFICANT IN-GAME STATISTICS AND DEVELOPING PREDICTION 57 MODELS FOR OUTCOMES OF NCAA DIVISION 1 FOOTBALL CHAMPIONSHIP

SUBDIVISION (FCS) GAMES

variable. The model was fit without an intercept term since the point spread should be zero if the in-game statistics were equivalent for both teams. Variables were then tested for significance, one at a time starting with the variable with the largest p-value, and taken out of the model if they were not significant at 0.05 . The final model arrived at using this technique was Model 3 with the parameter estimates given in Table 7 . It is noted that the home indicator variable was not included in this model. An attempt was made to put the home indicator variable into the model. It was not significant ( $p$-value $=0.9004)$ and the estimate of the coefficient was negative. This finding suggests that there is a relationship between the home indicator variable and some of the other variables already in the model.

Table 6

Odds Ratios for Model 2

\begin{tabular}{|cccc|}
\hline Effect & $\begin{array}{r}\text { Odds Ratio Estimates } \\
\text { Point Estimate }\end{array}$ & \multicolumn{2}{c|}{$\begin{array}{c}\text { 95\% Wald } \\
\text { Confidence Limits }\end{array}$} \\
\hline Difference_in_rank & 1.008 & 1.004 & 1.013 \\
TOM & 3.075 & 1.989 & 4.755 \\
diff_pass_comp & $>999.999$ & 29.892 & $>999.999$ \\
diff_sacks & 2.088 & 1.475 & 2.957 \\
diff_3rd_pct & 130.667 & 5.565 & $>999.999$ \\
diffpunt_return & 1.856 & 1.265 & 2.723 \\
diffave_yardsperplay & 1.572 & 1.044 & 2.365 \\
\hline
\end{tabular}

Table 7

Parameter Estimates for Model 3

\begin{tabular}{|cccccc|}
\hline Parameter & DF & Estimate & $\begin{array}{c}\text { Standard } \\
\text { Error }\end{array}$ & t Value & Pr $>|\mathbf{t}|$ \\
\hline TOM & $\mathbf{1}$ & 3.87225 & 0.28928 & 13.39 & $<.0001$ \\
diff_pen & $\mathbf{1}$ & -0.51624 & 0.14291 & -3.61 & 0.0004 \\
diff_first & $\mathbf{1}$ & 0.38999 & 0.08768 & 4.45 & $<.0001$ \\
diff_3rd_pct & $\mathbf{1}$ & 20.75532 & 3.39939 & 6.11 & $<.0001$ \\
diffpunt_return & $\mathbf{1}$ & 1.00627 & 0.4034 & 2.49 & 0.0133 \\
Diff_puntyards & $\mathbf{1}$ & 0.06932 & 0.02319 & 2.99 & 0.0031 \\
diffave_yardsperplay & $\mathbf{1}$ & 4.49276 & 0.34299 & 13.1 & $<.0001$ \\
\hline
\end{tabular}


Model 4 was formed by adding differences in ranks of the two teams playing to Model 3. Differences in rank was found to be significant ( $\mathrm{p}$-value $<0.0001$ ). The parameter estimates for Model 4 are given in Table 8 . The adjusted $\mathrm{R}^{2}$ value for this model was 0.8448 indicating that an estimated $84.48 \%$ of the variation in point spread of football games was explained by these variables.

Table 8

Parameter Estimates for Model 4

\begin{tabular}{|cccccc|}
\hline Parameter & DF & Estimate & $\begin{array}{c}\text { Standard } \\
\text { Error }\end{array}$ & t Value & Pr $>|\mathbf{t}|$ \\
\hline Difference_in_rank & $\mathbf{1}$ & 0.01975 & 0.00408 & 4.83 & $<.0001$ \\
TOM & $\mathbf{1}$ & 3.71258 & 0.27763 & 13.37 & $<.0001$ \\
diff_pen & $\mathbf{1}$ & -0.49136 & 0.13627 & -3.61 & $4 \mathrm{E}-04$ \\
diff_first & $\mathbf{1}$ & 0.33225 & 0.0844 & 3.94 & $1 \mathrm{E}-04$ \\
diff_3rd_pct & $\mathbf{1}$ & 19.68779 & 3.24687 & 6.06 & $<.0001$ \\
Diff_puntyards & $\mathbf{1}$ & 0.07278 & 0.02211 & 3.29 & 0.001 \\
diffpunt_return & $\mathbf{1}$ & 0.67187 & 0.39058 & 1.72 & 0.087 \\
diffave_yardsperplay & $\mathbf{1}$ & 4.13958 & 0.33491 & 12.36 & $<.0001$ \\
\hline
\end{tabular}

\section{Model Prediction and Accuracy}

We next wished to determine if we could use the four models we developed in order to predict the outcome of future football games. In this case, we will not know what the actual in-game statistics are ahead of time, or the actual differences of these in-game statistics, since the game has not been played. We considered the past three games that each team had played and explored various methods of estimating the differences of these in-game statistics for the future game we are trying to predict.

Data was obtained to test the accuracy of the four models for future predictions, by taking a stratified random sample of 52 games from the 2012 NCAA Division I FCS regular season. All thirteen conferences in the NCAA Division I FCS were considered and two teams were randomly sampled in each conference. Once the two teams were selected from each conference, we randomly sampled two games that each team was going to play in which to predict results. The games were randomly sampled between games 4 and 11 that the teams played since we needed three previous games that a team played during that season in order to estimate in-game statistics. There were no duplicate games included in the sample. The sampled games are given in Table 9.

The three games played by both teams prior to the game to be predicted were examined for both teams in order to estimate in-game statistics of the game to be predicted. Several methods were used to estimate the in-game statistics. These methods included the following: using the averages of all the in-game statistics from the last three games of both teams to estimate the in-game statistics for the team in the upcoming game; using the averages of all in-game statistics from the last two games of both teams; using the in-game statistics from 


\section{IDENTIFYING SIGNIFICANT IN-GAME STATISTICS AND DEVELOPING PREDICTION MODELS FOR OUTCOMES OF NCAA DIVISION 1 FOOTBALL CHAMPIONSHIP \\ SUBDIVISION (FCS) GAMES}

the last game played of both teams; using the median of the in-game statistics of the last three games to estimate the in-game statistics for the team in the upcoming game for both teams ; using the maximums of each of the last three in-game statistics for both teams; and using the minimum of each of the last three in-game statistics for both teams. Our final method in trying to estimate in-game statistics for the game we are trying to predict for was to consider the total yards that the team made during each of the last three games, find the game that had the median total yardage that the team made, and use the in-game statistics from this game. This last method ended up being our best attempt to estimate the future in-game statistics.

Table 9

Sampled Games from 2012 NCAA Division I FCS

\begin{tabular}{|lll|}
\hline Conference & 1st Team \& Games & 2nd Team \& Games \\
\hline Big Sky Conference & Idaho State - 5, 10 & Northern Colorado- 6, 11 \\
Big South Conference & Gardner-Webb- 4, 6 & VMI- 6, 8 \\
Colonial Athletic Association & Delaware- 9,11 & James Madison- 9, 10 \\
Ivy League & Columbia Lions- 6, 9 & Cornell- 7, 10 \\
Mid-Eastern Athletic Conference & Norfolk State- 8, 10 & North Carolina Central- 8, 11 \\
Missouri Valley Football Conference & Illinois State- 4, 5 & Missouri State- 5, 10 \\
Northeast Conference & Bryant University- 4, 8 & Wagner- 9, 10 \\
Ohio Valley Conference & Austin Peay- 7, 11 & Murray State- 4, 7 \\
Patriot League & Georgetown- 6, 7 & Holy Cross- 6, 11 \\
Pioneer Football League & Drake- 8, 10 & Morehead State- 4, 6 \\
Southern Conference & Citadel- 4,9 & Georgia Southern- 5, 7 \\
Southland Conference & Lamar- 5, 11 & McNeese State- 8, 11 \\
Southwestern Athletic Conference & Alabama A\&M- 5, 9 & Grambling State- 6,7 \\
\hline
\end{tabular}

As an example of using this method, we will use Model 3, and predict an outcome of a game played on November 3, 2012 where Missouri State University played North Dakota State University (Game 10 for both teams). We will first consider the total yardage that each of the teams made during their previous three games. The total yardages are given in Table 10 .

Table 10

Median Total Yards Example

\begin{tabular}{|r|c|c|}
\hline Game & MSU Total Yards & NDSU Total Yards \\
\hline 9 & 350 & 386 \\
8 & 305 & 385 \\
7 & 283 & 294 \\
\hline
\end{tabular}


After examining Table 10, you can see that the median total yardage of the last three games played by MSU was in game 8 . The in-game statistics from game 8 were used for MSU in estimating the in-game statistics for the upcoming game. The median total yardage for NDSU was also in game 8 and hence, the estimated in-game statistics for NDSU were their in-game statistics from game 8 . These in-game statistics for both of the teams are given in Table 11, and their difference in Table 12.

Table 11

In-game Statistics for MSU vs. NDSU

\begin{tabular}{|ccccccccc|}
\hline Team & Game & First & 3rd Down Pct & pen Turnovers & punt return punt yards & Average Yards Per Play \\
\hline MSU & 8 & 17 & $53.33 \%$ & 6 & 3 & 4 & 49 & 9.53 \\
NDSU & 8 & 21 & $57.14 \%$ & 7 & 0 & 4 & 132 & 9.87 \\
\hline
\end{tabular}

Table 12

Difference of In-game Statistics for MSU vs. NDSU (MSU-NDSU)

\begin{tabular}{|ccccccc|}
\hline Diff_first diff_3rd_pct & diff_pen & TOM & diffpunt_retum & diff_puntyards & diffave_yardsperplay \\
\hline-4 & $-3.81 \%$ & -1 & -3 & 0 & -83 & -1.24 \\
\hline
\end{tabular}

The difference in $3^{\text {rd }}$ down percentages was actually calculated in terms of differences in probabilities of getting a $3^{\text {rd }}$ down conversion. Therefore, the value of $-3.81 \%$ would be placed in the model as -.0381 . Now using Model 3 we obtain the following equation for predicting the point spread of this game:

Point Spread $=$ TOM $* 3.87225-$ diff_pen*0.51624 + diff_first*0.38999 + diff_ $3^{\text {rd }}$ pct*20.75532 $^{*}$ diffpunt_return*1.00627 + diff_puntyards*0.06932 + diffave_yardsperplay*4.49276

The estimated in-game statistics from the median total yards method were placed in the model and the point spread was estimated as follows:

Point Spread $=(-3) * 3.87225-(-1) * 0.51624+(-4) * 0.38999+(-0.0381) * 20.75532+(0) * 1.00627+$ $(-83) * 0.06932+(-1.24) * 4.49276=-24.77$

The predicted point spread for the game was -24.77 with NDSU winning the game. The actual score of the game was 17 to 21 with NDSU winning by 4 . The model correctly predicted a winning of the game, but overestimated the margin of victory.

All 52 games were predicted using each of the four models. We were interested only in whether the model predicted a win or loss for the "team of interest". If the estimated probability of the "team of interest" winning the game was higher than 0.50 using Models 1 and 2, we predicted a win for that team. Otherwise, we predicted a loss. If the estimated point spread was positive for the "team of interest" using Models 3 and 4, we predicted a win for that team. Otherwise, we predicted a loss. For this sample, the highest prediction accuracy was from Model 2 yielding and accuracy of 78.89\% which was the logistic regression model that used both in-game statistics and differences in ranks. The next most accurate model was Model 1 which was the logistic regression model that only considered in-game statistics yielding an accuracy of $75 \%$. Model 4 , the ordinary least squares regression model that included both in-game statistics and differences in ranks, yielded an accuracy of 73.08\%. The least accurate model was Model 3, which was the ordinary least squares method which only considered in-game statistics which yielded an accuracy of $69.23 \%$. The accuracy results are shown in Table 13 .

If one were to select the team playing at home as the team that was going to win, this method had an 


\section{IDENTIFYING SIGNIFICANT IN-GAME STATISTICS AND DEVELOPING PREDICTION MODELS FOR OUTCOMES OF NCAA DIVISION 1 FOOTBALL CHAMPIONSHIP \\ SUBDIVISION (FCS) GAMES}

accuracy of $67.31 \%$. If one used only the computer rankings of the teams to predict a winner, this method was correct $71.15 \%$ of the time. In comparison, Model 1 and Model 2 were $75 \%$ and $78.85 \%$ accurate for this sample. Three of our models had improved estimated improved prediction accuracies over using just the rankings, and all of the models had improved estimated prediction accuracies over just selecting the team playing at home.

Table 13

Accuracy Testing Results

\begin{tabular}{|cccc|}
\hline Models & Correct & Incorrect & Prediction Accuracy \\
\hline Model 1 & 39 & 13 & $75.00 \%$ \\
Model 2 & 41 & 11 & $78.85 \%$ \\
Model 3 & 36 & 16 & $69.23 \%$ \\
Model 4 & 38 & 14 & $73.08 \%$ \\
Computer Ranking & 37 & 15 & $71.15 \%$ \\
Home & 35 & 17 & $67.31 \%$ \\
\hline
\end{tabular}

\section{Conclusions}

When estimating the probability of a team winning the game using the logistic regression model in NCAA FCS Division 1 football, there were six differences of in-game statistics that were significant with the six statistics being: difference in number of turnovers; difference in probabilities of pass completions, difference in probabilities of a $3^{\text {rd }}$ down conversion, difference in number of defensive sacks, difference in number of punt returns, and difference in average number of offensive yards per play. When a least squares regression model was used to estimate the point spread, seven differences for in-game statistics were significant. These differences were the following: difference in number of turnovers; difference in number of penalties, difference in number of first downs, differences in number of punt returns, difference in number of punt return yards, difference in probabilities of third down completions, and difference in the average number of offensive yards per play. We estimated these in-game statistics differences based on the three previous games that each team played. When using these combined with the differences in team ratings as determined by Compughterratings.com in the week before a game is played, the estimated prediction accuracy over just using the difference in the teams' ratings increased. In logistic regression, this increased our estimated prediction accuracy by approximately $8 \%$. In the case of the least squares model, this increase our estimated prediction accuracy as to which team would win the game by approximately $2 \%$.

\begin{tabular}{|c|c|c|c|c|c|c|c|c|c|}
\hline \multicolumn{10}{|c|}{ References } \\
\hline \multicolumn{4}{|c|}{$\begin{array}{l}\text { Compughterratings.com. } \\
\text { http://www.compughterratings.com/CFB/ratings }\end{array}$} & February & 16 & 2013 & from & Comp & ratings.com: \\
\hline ESPN & Scoreboard. & (2013). & Retrieved & Feb & & 16 , & 2013 & from & ESPN.com: \\
\hline
\end{tabular}


Harville, D. (1977) The use of linear-model methodology to rate high school or college teams. Journal of the American Statistical Association, 72 (358): 278-289.

Magel, R. C., Childress, G. (2012). Examining the Outcome Effects of the Turnover Margin in Professional Football. International Journal of Sports Science and Engineering , 6 (3), 147-152

NCAA Stats. (2013). Retrieved February 16, 2013 from NCAA.org: http://web1.ncaa.org/stats/StatsSrv/rankings?doWhat=archive\&sportCode=MFB

Willoughby, K. A. (2002) Winning Games in Canadian Football: A Logistic Regression Analysis. The College Mathematics Journal 33 (3): 215-220. 